\title{
1 Untangling Microbiota Diversity and Assembly Patterns in 2 the World's Largest Water Diversion Canal
}

3 Lu Zhang ${ }^{1,2}$, Wei Yin ${ }^{3}$, Chao Wang ${ }^{3}$, Aijing Zhang ${ }^{4}$, Hong Zhang ${ }^{5}$, Tong Zhang ${ }^{6}$ and Feng Ju ${ }^{1,2^{*}}$

$4{ }^{1}$ Key Laboratory of Coastal Environment and Resources of Zhejiang Province, School of 5 Engineering, Westlake University, 18 Shilongshan Road, Hangzhou 310024, Zhejiang Province, 6 China

$7 \quad{ }^{2}$ Institute of Advanced Technology, Westlake Institute for Advanced Study, 18 Shilongshan Road,

8 Hangzhou 310024, Zhejiang Province, China

$9{ }^{3}$ Changjiang Water Resources Protection Institute, 515 Qintai Street, Wuhan 430051, Hubei 10 Province, China

$11{ }^{4}$ Construction and Administration Bureau of South-to-North Water Diversion Middle Route 12 Project, 1 Yuyuantan South Road, Beijing 100038, China

135 State Key Laboratory of Environmental Aquatic Chemistry, Research Center for 14 Eco-Environmental Sciences, Chinese Academy of Sciences, 18 Shuangqing Road, Beijing 15 100085, China

$16{ }^{6}$ Environmental Microbiome Engineering and Biotechnology Laboratory, Department of Civil 17 Engineering, Pokfulam Road, The University of Hong Kong, Hong Kong 999077, China

* Corresponding to:

Dr. Feng Ju (Assistant Professor)

21 Address:

22 Environmental Microbiome and Biotechnology Lab (EMBLab)

23 Westlake University, 18 Shilongshan Road, Hangzhou 310024, China

24 Tel.: 571-87963205 (lab), 571-87380995 (office)

25 Fax: 0571-85271986

26 E-mail: jufeng@westlake.edu.cn 


\section{Abstract}

29 Large water diversion projects are important constructions for reallocation of human-essential

30 water resources. Deciphering microbiota dynamics and assembly mechanisms underlying

31 canal water ecosystem services especially during long-distance diversion is the prerequisite

32 for water quality monitoring, biohazard warning and sustainable management. Using a

33 1432-km canal of the South-to-North Water Diversion Projects as a model system, we answer

34 three central questions: how bacterial and micro-eukaryotic communities spatio-temporally

35 develop, how much ecological stochasticity contributes to microbiota assembly, and which

36 immigrating populations better survive and navigate across the canal. We applied quantitative

37 ribosomal RNA gene sequence analyses to investigate canal water microbial communities

38 sampled over a year, as well as null model- and neutral model-based approaches to

39 disentangle the microbiota assembly processes. Our results showed clear microbiota dynamics

40 in community composition driven by seasonality more than geographic location, and

41 seasonally dependent influence of environmental parameters. Overall, bacterial community

42 was largely shaped by deterministic processes, whereas stochasticity dominated

43 micro-eukaryotic community assembly. We defined a local growth factor (LGF) and

44 demonstrated its innovative use to quantitatively infer microbial proliferation, unraveling

45 taxonomically dependent population response to local environmental selection across canal

46 sections. Using LGF as a quantitative indicator of immigrating capacities, we also found that

47 most micro-eukaryotic populations $(82 \%)$ from the source lake water sustained growth in the

48 canal and better acclimated to the hydrodynamical water environment than bacteria $(67 \%)$. 
49 Taxa inferred to largely propagate include Limnohabitans sp. and Cryptophyceae, potentially

50 contributing to water auto-purification. Combined, our work poses first and unique insights

51 into the microbiota assembly patterns and dynamics in the world's largest water diversion

52 canal, providing important ecological knowledge for long-term sustainable water quality

53 maintenance in such a giant engineered system.

54 Key words: Engineered system; Bacterial community; Micro-eukaryote; Deterministic

55 assembly; Stochastic assembly; Population growth

56 


\section{Introduction}

58 Microorganisms are fundamental components of ecosystems and play vital roles in global

59 biogeochemical cycling (Arrigo, 2005; Battin et al., 2016; Du et al., 2021). Knowledge about

60 composition and dynamics of microbial communities across space and time is a prerequisite

61 for predicting and manipulating the microorganisms in both natural and engineered systems

62 (Bell et al., 2005; Bru et al., 2011; Ju and Zhang, 2015). Due to artificial system design and

63 control, microbial communities in engineered ecosystems have received increasing attention

64 to unravel the community dynamics underlying their eco-environmental or biotechnological

65 services (Briones and Raskin, 2003; Ju et al., 2017; Ju et al., 2014; Vanwonterghem et al.,

66 2014; Wu et al., 2019). Specifically, microorganisms in drinking water systems are closely

67 linked with water auto-purification and eventually to public health (Berry et al., 2006). Thus,

68 comprehensive microbiome monitoring is needed to differentiate baseline and disturbed

69 microbial communities in engineered water systems to facilitate system management (Hull et

70 al., 2019; Sagova-Mareckova et al., 2021).

72 in the drought-stricken north China, which represent the world's largest water diversion

73 projects. The projects consist of the Eastern Route Project, the Middle Route Project (MRP)

74 and the Western Route Project. The 1432-km MRP went into service in 2014 and has drawn

7540 billion $\mathrm{m}^{3}$ of water from the Danjiangkou Reservoir to He'nan and Hebei Provinces, and

76 Beijing and Tianjin Municipalities, benefiting tens of millions of people. Despite their 
77 important roles in controlling geochemical processes and indicating water quality (Chen et al.,

78 2018; Dang et al., 2018), little has been known about the microbial constituents throughout

79 the system (Luo et al., 2019), neither for other engineered long-distance water diversion

80 canals. Especially, the MRP is a closed engineered system where microorganisms are subject

81 to potentially less complex biotic and abiotic interactions, while the numerous hydraulic

82 structures and engineered perturbations (e.g., inverted siphon, aqueduct and tunnels) along the

83 main canal add to challenges to model microbial community dynamics, calling for

84 comprehensive investigation of microbial community patterns in the MRP canal, to assist in

85 establishing integrative assessment of water quality with microbial community monitoring

86 (Sagova-Mareckova et al., 2021).

87 Understanding processes and mechanisms driving the community patterns underlying

88 ecosystem services is one of the key issues in microbial ecology (Zhou et al., 2014).

89 Microbial community composition in aquatic ecosystems can be affected deterministically by

90 both abiotic or environmental factors, e.g., water temperature, $\mathrm{pH}$, dissolved oxygen, and

91 nutrient availability (Hu et al., 2017; Liu et al., 2020a; Zhang et al., 2019b), and biotic factors,

92 such as competition and predation. In addition to deterministic mechanisms, stochastic

93 processes, for instance, dispersal and ecological drift, also contribute to microbial community

94 assembly. Efforts have been made to disentangle the relative importance of deterministic vs.

95 stochastic processes based on a variety of null and neutral modeling methods (Ning et al.,

96 2019; Sloan et al., 2006; Stegen et al., 2013). Depending on which ecosystems or organism

97 types these methods are applied to, the relative importance of deterministic vs. stochastic 
98 processes may greatly vary (Chen et al., 2019; Liu et al., 2018b; Wang et al., 2020; Yan et al.,

99 2020; Niederdorfer et al., 2021). For instance, bacterial communities in lake water were

100 revealed to be predominantly structured by determinism, whereas micro-eukaryotic

101 communities largely by stochasticity (Logares et al., 2018). For the MRP, the closed channel,

102 the environmental gradients and the variety of hydraulic constructions along the canal make it

103 obscure to what extent ecological stochasticity determines the assembly of microbiota

104 community, which is essential for an improved understanding of the ecosystem processes and

105 microbial dynamics prediction.

Other emerging questions in such large water-diversion canal and the comparable

107 systems, like river continuum (Gweon et al., 2021), include which immigrating

108 microorganisms are adapted to local niches in the receiving water and how they functionally

109 contribute to the ecological service. Especially in the MRP, how many of the microbial

110 populations originated from a natural reservoir may survive in the contrastingly engineered

111 canal remains an open question. Although population growth associated with microbial

112 immigration has been quantified in other engineered water systems (Mei and Liu, 2019), such

113 as activated sludge reactors (Mei et al., 2019), efforts in estimating microbial growth in

114 long-distance and evolving systems are still lacking.

115 In the present study, we followed the spatio-temporal dynamics of prokaryotic and 116 micro-eukaryotic community along the main canal of MRP of the SNWD project over a year 117 to address: 1) How does microbial community develop with the water flow? 2) How is the 
118 relative importance of deterministic and stochastic processes on shaping the community

119 assembly in this closed turbulent engineered system? 3) How do the immigrating bacteria and

120 micro-eukaryotes from water source survive, grow, or die off in the canal? Based on

121 high-throughput marker gene (i.e., 16S and 18S) amplicon and metagenomic sequencing,

122 bioinformatics, and statistical modeling of water microbiota, we elucidated the microbial

123 diversity and population dynamics along the canal over four seasons and uncovered the key

124 patterns and processes driving community assembly. By overcoming the limitations of widely

125 used relative abundance measures with estimated absolute abundance (Barlow et al., 2020;

126 Tkacz et al., 2018), we demonstrated the first use of local growth factor to predict growth

127 capacity of the immigrating microbial populations along the water canal and revealed the

128 divergent microbial fates and population lifestyles throughout the world's largest water

129 diversion canal.

\section{Materials and Methods}

131 A full version of the Materials and methods are available in the Supplementary Information.

Study sites, sampling and physiochemical monitoring

133 The Middle Route Project (MRP) of the South-to-North Water Diversion (SNWD) Projects

134 started from Danjiangkou reservoir and diverted water northwards until Beijing and Tianjin,

135 which is an artificial system without direct connection with surface waters in the area. Fifty

136 samples were collected from 19 sites (Fig. 1), including one at the Danjiangkou reservoir

137 (S01) and 18 along the main canal of the MRP (S02-S18). The 18 sampling sites along the 
138 canal were selected to capture the spatial dynamics of microbial communities from canal head

139 to end across the subtropical and warm-temperate climate zones. The sites were grouped to

140 three sections: S02-S08 (Section1), S09p-S14 (Section2) and S15-S18 (Section3). Canal

141 water was sampled and filtered through $0.22-\mu \mathrm{m}$ cellulose nitrate filters (Sartorius, Germany).

142 Physiochemical properties, including water temperature (T), pH, dissolved oxygen (DO), total

143 nitrogen $(\mathrm{TN})$ and fluoride $\left(\mathrm{F}^{-}\right)$, were measured according to the environmental quality

144 standard for surface water of China (GB3838-2002). Details about the sampling sites and

145 physiochemical measurements are in the Supplementary Method S1.

\section{DNA extraction, PCR amplification and sequencing}

147 Genomic DNA was extracted from filters using FastDNA Spin Kit for Soil (MP Biomedicals,

148 USA). Bacterial 16S rRNA gene V3-V4 region and eukaryotic 18S rRNA gene V4 region

149 were amplified and sequenced on the Illumina platforms producing 250 bp paired-end reads at

150 the Guangdong Magigene Biotechnology Co., Ltd. (Guangzhou, China). Real-time

151 quantitative PCR (qPCR) was additionally performed to quantify the 16S rRNA gene copies

152 (as a proxy for bacterial biomass) in each genomic DNA sample, as described in Ju et al.

153 (2019). Detailed methodologies are provided in the Supplementary Method S2.

154 Each genomic DNA was also used for shot-gun metagenomic sequencing on the 155 Illumina's Novaseq platform using a 150 bp paired-end sequencing strategy at the Novogene 156 (Beijing, China). The sequencing data generated in this study have been deposited into CNGB

157 Sequence Archive (CNSA) (Guo et al., 2020) of China National GeneBank DataBase 
158 (CNGBdb) (Chen et al., 2020) with accession number CNP0001593.

\section{Bioinformatics and statistical analyses of microbial diversity}

160 The 16S and 18S rRNA gene amplicon sequence data were processed using Qiime2 pipeline

161 (Bolyen et al., 2019) and DADA2 algorithm (Callahan et al., 2016) as described in the

162 Supplementary Method S3, generating final abundance tables of amplicon sequence variants

163 (ASVs) consisting of 6705 bacterial and 3217 eukaryotic ASVs, respectively. The

164 alpha-diversity metrics of bacterial and micro-eukaryotic communities were further computed

165 in Qiime2. All the univariate and multivariate statistics were performed in R (R Core Team,

166 2020). A set of spatial factors was generated for the sampling sites based on the geographic

167 coordinates using the principal coordinates of neighboring matrices (PCNM) analysis

168 (Borcard and Legendre, 2002).

169 The metagenomic data were pretreated and searched against the SILVA SSU132 database

170 to identify rRNA gene reads. This information was further used to estimate the 16S rRNA and

171 18S rRNA gene copies detected in each metagenome based on the sum for the read coverage

172 over the full length of each matched reference sequence in the database. Equation and details

173 are available in the Supplementary Method S3. The copies of 18S rRNA genes in each water

174 sample were estimated as 16S rRNA gene copies (determined by the aforementioned qPCR

175 analysis) divided by ratios of $16 \mathrm{~S}$ rRNA to $18 \mathrm{~S}$ rRNA gene copy (Table S1) as sequenced and

176 estimated in the metagenomic data. 


\section{Neutral and null model analyses of microbial community patterns}

178 To disentangle the relative contribution of stochastic and deterministic processes to microbial

179 community assembly, Sloan neutral model (Sloan et al., 2006) and null model-based NST

180 approach (Ning et al., 2019) were applied to the $16 \mathrm{~S}$ and $18 \mathrm{~S}$ community data. Fitting of the

181 model were performed in $\mathrm{R}$ according to Burns et al. (2016). Accordingly, ASVs were

182 classified into three groups depending on whether their observed occurrence falling higher

183 than ("above prediction"), within ("as predicted") or lower than ("below prediction") the 95\%

184 confidence interval of the neutral model predictions. Normalized stochasticity ratio (NST)

185 was used to quantify ecological stochasticity in communities within canal sections (Ning et al.,

186 2019). NST analysis was performed in R using the NST package (Ning et al., 2019). More

187 details about the methodology are in the Supplementary Method S4.

188 Population lifestyle and local growth factor analyses

189 The absolute abundance of bacterial and micro-eukaryotic ASVs was calculated by

190 multiplying relative abundance of each ASV computed from amplicon sequence data by the

191 overall $16 \mathrm{~S}$ and $18 \mathrm{~S}$ rRNA gene copies detected in a given metagenome for all the Oct

192 samples (see the Supplementary Method S5 for more details).

193 To characterize fate of microorganisms originated from natural reservoir after transported

194 into the closed canal, we defined a local growth factor (LGF) to depict whether the

195 populations grew or restrained, by comparing their mean absolute abundance in each canal 
section to the abundance at the canal head, as follows:

$$
\mathrm{LGF}_{\mathrm{x}}=\log _{10} \frac{\mathrm{AA}_{\mathrm{x}, \mathrm{N}}+1}{\mathrm{AA}_{\mathrm{x}, \mathrm{OCtSO2}+1}}
$$

where $\mathrm{AA}_{\mathrm{x}, \mathrm{N}}$ is the average absolute abundance of ASV $\mathrm{x}$ in Section $\mathrm{N}$ of sampling sites and

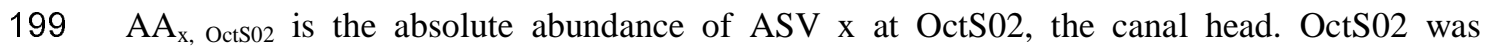

200 excluded from Section1 for the LGF calculation. An average LGF > 0 across the three

201 sections was considered to indicate growth in the canal. The potentially pathogenic bacterial

202 ASVs were identified referring to a list of potentially pathogenic genera (Table S2)

203 summarized from Woolhouse et al. (2015), and LGFs of these ASVs were examined.

\section{Results}

\section{Microbiota biomass and diversity shifted with seasonality}

206 Microbial communities showed clear seasonal shifts (Fig. 2), whether for their biomass (A

207 and B) or alpha (C-F) and beta diversity (Fig. 2G, 2H and Fig. S1). Both the bacterial and

208 micro-eukaryotic biomass, as measured by 16S rRNA (A) and 18S rRNA (B) gene copies,

209 were the highest in August and the lowest in October (Fig. 2). PERMANOVA showed that

210 seasonality significantly $(P<0.001)$ influenced both the bacterial $(F=22.78)$ and

211 micro-eukaryotic $(F=25.57)$ community composition. Moreover, the seasonal impacts were

212 much more pronounced than the spatial effects along the canal, whether for bacteria $\left(R^{2}=\right.$

2130.52 vs. 0.14$)$ or micro-eukaryotes $\left(R^{2}=0.55\right.$ vs. 0.14). Accordingly, Bray Curtis-based

214 NMDS analyses of all canal water samples $(n=52)$ revealed strong clustering of bacterial 
215 (Fig. 2G) and micro-eukaryotic (Fig. 2H) communities in relation to seasons, of which May

216 and August had witnessed greater intra-season community variations along the canal.

217 Seasonal variation was also observed for specific bacterial and micro-eukaryotic lineages (Fig.

218 S2 and S3).

219 Microbiota composition and diversity changed along the canal

220 Both the source water from Danjiangkou reservoir (S01) and the canal water were dominated

221 by phyla Actinobacteria (39.7 - 65.0\%, mainly hgcI clade and CL500-29 marine group),

222 Proteobacteria (17.9 - 38.3\%), Bacteroidetes (0.9 - 29.5\%), Verrucomicrobia $(1.2-9.2 \%)$ and

223 Cyanobacteria (0.2 - 8.6\%, Fig. 3A). Overall, bacterial communities at the canal head (S02)

224 were most structurally similar to the source water (Fig. 3A and Fig. S1A). However,

225 compared with the source water, relative abundance of Proteobacteria at the canal head (i.e.,

226 S02) decreased whereas Actinobacteria and Bacteroidetes increased. Within the canal, both

227 Cyanobacteria (mostly Cyanobium_PCC-6307, $F=16.77, P<0.001)$ and Bacteroidetes $(F=$

$2286.06, P<0.05$ ) showed significant decrease in relative abundance with water flow (Fig. 3A).

229 In contrast, Limnohabitans $(F=19.87)$ and Candidatus Methylopumilus $(F=58.69)$ within

230 Proteobacteria became increasingly abundant along the canal $(P<0.001)$. Likewise, both the

231 overall bacterial biomass (Fig. S4A) and Chao1 richness (Fig. S5A) exhibited highly dynamic

232 variation patterns along the canal. The variation trend of Shannon diversity index largely

233 agreed with (though relatively weaker than) that of Chao1 richness (Fig. S5B).

234 Unlike bacterial communities, micro-eukaryotic communities appeared more dynamic 
235 along the canal water flow (Fig. 3B). The relative abundance of Metazoa, mostly Calanoida,

236 was as high as $24.0 \%$ in Danjiangkou Reservoir. These organisms were drastically enriched

237 by 2.3 times upon entering the canal head site, but largely declined in the downstream sites

238 (S03 to S18). Two predominant lineages of Cryptophyceae, i.e., Cryptomonas $(F=4.11, P=$

$2390.06)$ and Teleaulax $(F=9.50, P<0.01)$, were more abundant in the source water and canal

240 upstream (S02-S08) than downstream sites (S09p-S14), accompanied by their gradual

241 replacement by two uncultured Cryptophyceae lineages $(F=30.53, P<0.001 ; F=24.60, P<$

2420.001 , respectively), especially close to the canal end (S15-S18). The canal head communities

243 were distinct from water source and the downstream communities (Fig. 3B and Fig. S1B).

244 This special pattern was manifested by the dominance of Calanoida $(72.0 \%)$ at the canal head

245 site where micro-eukaryotic biomass was much higher $\left(3.2 \times 10^{9}\right.$ vs. average $5.9 \times 10^{8} 18 \mathrm{~S}$

246 rRNA gene copies $\mathrm{L}^{-1}$, Fig. S4B) whereas alpha diversity metrics much lower compared with

247 the other sites (Fig. S6), suggesting intriguing local micro-eukaryotic selection in the canal

248 head.

249 Environmental factors influenced canal microbiota diversity and biomass

250 The influence of five environmental parameters, namely water temperature, $\mathrm{pH}$, dissolved

251 oxygen (DO), total nitrogen (TN) and fluoride (Table S3), and spatial factors (i.e., ten PCNM

252 variables representing the quantitative spatial patterns, Table S4) on the microbial

253 communities was tested by redundancy analysis. All the above-mentioned environmental

254 variables and PCNM1, 2 and 4 were identified to exert significant impacts on bacterial 
255 community composition $(P<0.05$, Fig. 4A). These variables except for PCNM4 also

256 represented the significant influential factors for micro-eukaryotic communities $(P<0.05$; Fig.

257 4B). Seasonal variation of environmental factors appeared based on the RDA plots (Fig. 4A

258 and 4B), for instance, fluoride concentration was especially higher in March, which was

259 supported by the significant seasonal difference in the environmental variables by

260 Kruskal-Wallis one-way ANOVA (Table S5). The abundant actinobacterial hgcI clade $\left(\mathrm{r}_{\mathrm{s}}=\right.$

$2610.63, P<0.01)$ and CL500-29 $\left(\mathrm{r}_{\mathrm{s}}=-0.68, P<0.01\right)$ marine group were among the bacterial

262 lineages positively and negatively related to fluoride concentration, respectively (Table S6).

263 For micro-eukaryotes, for instance, an ASV affiliated to Mediophyceae $\left(\mathrm{r}_{\mathrm{s}}=0.66, P<0.01\right)$

264 was positively correlated with fluoride (Table S7).

265 The identified significant variables were employed in further variation partitioning 266 analysis (VPA). The result showed clearly stronger influence of seasonality than that of 267 spatial and examined environmental factors for both bacterial and eukaryotic communities

268 (Figure 4C and 4D). Seasonality alone could explain 19\% and $17 \%$ of the bacterial and

269 micro-eukaryotic community variance, respectively, while only $6-9 \%$ of the variance could

270 be explained exclusively by environmental or spatial factors. Notably high proportions of the

271 community variance could be attributed to the co-effects of seasonality and environmental

272 factors (29\% for bacteria and $23 \%$ for micro-eukaryotes), implying their strong interplay in

273 shaping microbial communities. Besides, temperature, DO and TN were found to be

274 significantly correlated with bacterial biomass, while only DO for micro-eukaryotic biomass

275 (as measured by 16S rRNA and 18S rRNA gene copies, Table S8). 

dynamic stochasticity

278 The apparent dispersal of microorganisms in the canal water and engineered hydraulic

279 manipulations (i.e., inverted siphon, aqueduct, and tunnels) along the main canal drove us to

280 question on the relative contribution of stochastic vs. deterministic processes to the canal

281 bacterial and micro-eukaryotic community assembly, which was first quantitatively assessed

282 with a recently proposed metric, normalized stochasticity ratio (NST) (Ning et al., 2019). This

283 new metric quantifies the relative importance of stochasticity in governing community

284 assembly ranging from 0 to 1 , with a value $>0.5$ indicating more stochastic assembly and <

2850.5 more deterministic assembly. NST analysis revealed contrasting trends between bacteria

286 and micro-eukaryotes. In the upstream section, a highest NST of 0.78 was estimated for

287 bacteria (Fig. 5A). This value decreased to 0.46 in the middle section, and to as low as 0.32

288 close to the canal end. On the contrary, NST was generally above 0.5 for micro-eukaryotes

289 throughout the canal, with the highest value of 0.75 in the middle section (Fig. 5A).

While NST provides rigorous quantification of relative importance of stochasticity, the

291 Sloan neutral model analysis (Sloan et al., 2006) allows for assessing how well the observed

292 community patterns could be explained by neutral processes (e.g., birth, death, and

293 immigration) and attributing the observed community-level assembly pattern to the divergent

294 population behavior. According to seasonal comparison of the model fitness, neutral model

295 could explain much higher proportions of community patterns in October $\left(R^{2}=0.73\right.$ and 0.67 
296 for bacteria and micro-eukaryotes, respectively) and March $\left(\mathrm{R}^{2}=0.77\right.$ and 0.72$)$, but lowest 297 in May $\left(\mathrm{R}^{2}=0.42\right.$ and 0.13 , Fig. S7). micro-eukaryotic ASVs were classified into three groups based on the neutral model analysis

300 of the October samples. Depending on whether they empirically occurred more frequently

301 than, as frequently as or less than predicted by the neutral model, ASVs were assigned to 302 "above prediction", "as predicted" and "below prediction" groups that were different in 303 abundance and composition (Fig. 5B-D). We found that $7.8 \%$ of the bacterial community 304 richness (i.e., number of ASVs) accounted by above-prediction group corresponded to $67.9 \%$ 305 of the total number of sequences, which was dominated by Actinobacteria and Proteobacteria, 306 whereas the neutrally distributed group contributed $86.8 \%$ of the richness but only $23.9 \%$ of 307 the abundance (Fig. 5B and Fig. S8). Similar trend was detected for micro-eukaryotes (Fig. 308 5B). The three partitioning groups of ASVs also showed distinct taxonomic composition (Fig. 309 5C, 5D and Fig. S8). Cryptomonas constituted 13.7\% of the above-prediction group in 310 abundance, but they were almost absent in the neutral and below-prediction groups. Metazoa, 311 in contrast, accounted for only $0.6 \%$ of the above-prediction group, but as high as $32.9 \%$ and $31262.3 \%$ of the abundance of the neutral and below-prediction groups (Fig. 5D).

313 Selective growth of immigrating bacterial and micro-eukaryotic populations along the 314 canal

315 To resolve which populations of the water communities died off, sustained, or proliferated 
316 during dispersal over 1432-km canal, we proposed local growth factor (LGF) (see equation 1

317 in Methods) as a quantitative measure of variation in population abundance in each canal

318 section compared to the abundance at the canal head, with an average LGF $>0$ across the

319 three sections indicating growth in the canal. LGF analysis was applied with the Oct sample

320 dataset composed of 1389 bacterial and 1229 eukaryotic ASVs, using absolute abundance of

321 ASVs estimated by multiplying relative abundance of ASVs at each canal site with the total

322 copies of bacterial $16 \mathrm{~S}$ rRNA $\left(7.89 \times 10^{8}\right.$ to $2.93 \times 10^{9}$ copies $\left.\mathrm{L}^{-1}\right)$ or micro-eukaryotic $18 \mathrm{~S}$

323 rRNA $\left(3.62 \times 10^{8}\right.$ to $3.19 \times 10^{9}$ copies $\left.\mathrm{L}^{-1}\right)$ genes therein. Micro-eukaryotes showed higher

324 overall occurrence $(0.313 \pm 0.008$ vs. $0.216 \pm 0.007 ; P<0.001)$ while lower variability

$325(2.879 \pm 0.033$ vs. $3.055 \pm 0.032 ; P<0.001)$ in absolute abundance compared to bacteria.

326 In total, $67.1 \%$ of the bacterial ASVs (Table S9) and $82.3 \%$ of the micro-eukaryotic

327 ASVs (Table S10) displayed positive average LGFs. The ASVs were further observed to form

328 clearly three clusters based on their LGFs in each section: LGF $>3,-3<$ LGF $<3$ and LGF $<$

$329-3$, which could indicate substantial growth, survival, and die-off of the microbial populations

330 (Fig. 6). The survival group represented the largest proportion of the ASVs (45.6 - 60.2\%),

331 while only $4.1-21.7 \%$ of the ASVs went extinction in the canal. We also found that both the

332 contrastingly high and low LGFs tended to be attributed to high-abundance microbial lineages

333 (Fig. 6A and B). For example, the abundant Luteolibacter sp. (ASV106) and Limnohabitans

334 sp. (ASV27) exhibited the highest LGF values in Section 2 and Section 3, respectively (Table

335 S9). In stark contrast, ASV138 assigned to actinobacterial CL500-29 marine group and two

336 Cyanobium PCC-6307 lineages (ASV144 and ASV250) were especially restrained in growth 
337 close to the canal end (Table S9). A total of 53 bacterial ASVs in the canal were related to 16

338 genera included in a published reference pathogen list (Table S2, summarized from

339 Woolhouse et al., 2015). While LGF of most (39 out of 53) of these potentially pathogenic

340 ASVs fell between -3 and 3, three Acinetobacter-associated ASVs (ASV794, ASV1108 and

341 ASV 1261) showed LGFs ranging from -5.9 to -6.1, indicating notable depletion along the

342 canal. For micro-eukaryotes, two uncultured Cryptophyceae lineages (ASV54 and ASV80) as

343 well as two ASVs related to Acineta flava (ASV224) and Spumella sp. (ASV59) were

344 elevated throughout the canal (Table S10).

\section{Discussion}

346 Diversion canals widely built for reallocation of water resources represent an ideal engineered

347 system to model microbial communities fulfilling key eco-environmental services, as well as

348 reflecting changes in ecosystem structure and function. Based on quantitative metagenomic

349 approaches that enable absolute quantification of microbial taxon, we uncovered the

350 microbiota spatio-temporal dynamics, key processes driving community assembly, and

351 navigation of immigrating microorganisms through the world's largest water diversion

352 project.

\section{Microbiota composition and biomass are dynamic along the canal}

354 Both bacterial and micro-eukaryotic communities in the source water showed marked

355 variations in taxonomic composition upon entering the canal, which could be attributed to the 
356 readily comprehensible differences in the environmental conditions between the natural

357 reservoir and its receiving engineered canal and potential influences exerted by the approach

358 channel through which source water enters the canal. Meanwhile, we observed intriguingly

359 decreased water microbial diversity after water transportation through an inverted siphon (S09

360 vs. S09p, Fig. S5 and S6). Taken together, these suggest that hydraulic structures along the

361 canal apply considerable influence on the microbial community diversity and composition,

362 which needs to be fully considered when setting baseline communities for long-term

363 microbial monitoring in the water diversion canal.

364 The prevalence of uncultured actinobacterial hgcI clade and CL500-29 marine group

365 echoed what was previously reported in other freshwater habitats (Keshri et al., 2018; Yang et

366 al., 2017). While both groups of yet-to-be-cultured lineages have been shown to favor lower

367 level of dissolved organic carbon and temperature (Lindh et al., 2015), the hgcI clade was

368 additionally implicated to be competitively advantageous in utilizing low-concentration

369 organic carbon (Glöckner et al., 2000) and to harbor potentially photoheterotrophic species

370 showing preferential utilization of nitrogen-rich organic compounds in freshwater (Ghylin et

371 al., 2014), implying its potential role in the nutrient uptake and water auto-purification.

372 Unlike bacterial communities, micro-eukaryotic communities in the canal have not yet

373 been explored before. By virtue of the high-throughput molecular analyses, we detected not

374 only Rotifea, copepods and protozoa previously microscopically observed as abundant in the

375 Danjiangkou Reservoir (Li et al., 2012), but also thousands of micro-eukaryotic populations 
376 (as ASVs), of which $6.7 \%$ are uncultured or unclassified even at the class level, implying

377 detection of numerous novel populations in the canal water. Notably, metazoan Calanoida

378 was repeatedly found to show drastic increase in abundance in the micro-eukaryotic

379 communities of canal head, compared with in the source water in both October and August.

380 This big order of aquatic crustaceans belonging to Copepoda inhabiting marine and

381 freshwater environments are found as the most successful among zooplankton colonizing

382 pelagic habitats (Blanco-Bercial et al., 2011; Bradford-Grieve, 2002). We further noted that

383 the SAR clade and Cryptophyceae showed substantially decreased relative abundance as a

384 result of high enrichment of Calanoida compared with the source water. However, their

385 absolute abundance or biomass, as we estimated as their 16S rRNA gene copies, remained

386 comparable $\left(2.78\right.$ vs. $3.43 \times 10^{8} 16 \mathrm{~S}$-copies $\mathrm{L}^{-1}$ for the SAR clade, and 3.68 vs. $4.25 \times 10^{8}$

387 16S-copies $\mathrm{L}^{-1}$ for Cryptophyceae). This strong decoupling pattern between relative and

388 absolute abundance cannot have been resolved by currently popular sequence-based relative

389 measures alone which have been shown to provide evidently misleading information on the

390 variation of absolute abundance (Stämmler et al., 2016; Vieira-Silva et al., 2019). It also well

391 demonstrates the power of our quantitative metagenomics approaches as an alternative to a

392 more direct but difficult-to-achieve quantitative PCR assay approach (due to the lack of $18 \mathrm{~S}$

393 rRNA gene universal primers) for estimating total micro-eukaryotic biomass. 
394 Microbiota assembly is deterministically driven by seasonally dependent habitat 395 filtering effects

396 Elucidating mechanisms and factors shaping biogeographic patterns has become a central

397 goal in microbial ecology (Hanson et al., 2012). Environment exerts influence on community

398 composition by selecting for populations that better adapted to the corresponding ecological

399 niches. We found that temperature, $\mathrm{pH}$, dissolved oxygen, total nitrogen and fluoride all

400 showed significant effects on the community dynamics in the main canal. Fluoride affects

401 bacterial growth and metabolic activity through targeting enzyme inhibition (Liao et al., 2015).

402 Fluoride has been reported to alter microbial community in high-fluoride groundwater (Zhang

403 et al., 2019a) and to exhibit inhibitory effect on bacterial populations involved in various

404 wastewater treatment processes (Ochoa-Herrera et al., 2009). Our results showed that fluoride

405 with concentration falling within the range of $0.14-0.22 \mathrm{mg} \mathrm{L}^{-1}$ (Table S3) could also affect

406 microbial community structure in a drinking water diversion system, where fluoride could be

407 mainly contributed by natural processes, such as mineral weathering (Nong et al., 2019), and

408 atmospheric pollutants (Pu et al., 2017). Interestingly, we found positive response of the hgcI

409 clade to elevated fluoride concentration (Table S6). While the mechanisms of their fluoride

410 resistance still await exploration, such evidence is in line with their favorable distribution in a

411 wide range of water ecosystems.

412 Although bacterial and micro-eukaryotic communities are both significantly influenced

413 by the examined environmental variables and spatial segregation, their overall variation were 
414 explained more by seasonality. Meanwhile, the strong co-influence of temporal and

415 environmental factors revealed by variation partitioning analysis could be at least partially

416 explained by seasonal variation of the examined variables (Table S5), suggesting

417 season-driven habitat filtering effects on microbiota. These results together imply a strong

418 interplay between temporal, spatial and environmental factors on influencing microbial

419 communities in the canal water, as previously noticed in natural lakes (Hu et al., 2017).

420 Although potential contribution from unmeasured environmental factors cannot be ruled out,

421 the remaining unexplained variation could possibly be generalized and linked to the influence

422 of stochastic processes.

423 Contrasting proportion of stochasticity contributes to bacterial and micro-eukaryotic

424 community assembly

425 Microbial community assembly is typically governed by four processes: selection, drift,

426 speciation and dispersal (Vellend and Agrawal, 2010). While selection is inherently

427 deterministic and drift is stochastic, speciation and dispersal could involve both deterministic

428 and stochastic mechanisms (Chase and Myers, 2011). For the MRP main canal, an

429 investigation period of one year is probably short for resolving speciation. Thus, selection

430 (deterministic processes), drift (stochastic processes) and dispersal (involving both) are

431 expected to shape the observed community assembly patterns, and their relative contributions

432 could be disentangled to help answer when, where, why and how much each process

433 contribute to the observed patterns (Hanson et al., 2012). 
434 Based on NST estimation and neutral modeling, we found that relative importance of

435 stochastic and deterministic processes in microbial community assembly differ between

436 bacteria and micro-eukaryotes, and among seasons and the canal sections. Neutral processes

437 explained $\sim 67 \%$ to $76 \%$ of the community patterns in October and March, but notably less in

438 May ( 41\% and 13\% for bacteria and eukaryotes, respectively). Liu et al. (2018a) reported a

439 prominent shortage in water storage of Danjiangkou Reservoir related to seasonal flood

440 control in May, which could have possibly imposed higher environmental selection effect to

441 the microbiota in the reservoir and across the canal, making the observed decreased

442 community stochasticity (or increased determinism) plausible. Although ecological

443 stochasticity adds to challenge to predict microbial communities, the new knowledge

444 produced in this study undoubtedly contribute to an improved understanding on when the

445 canal water microbiota is more prone to stochastic processes, or under which conditions

446 stochasticity is of more relevance.

The NST values suggested that the relative contribution of deterministic processes is

448 larger to bacterial community assembly compared with micro-eukaryotic communities, where

449 stochasticity prevails. Recently, Gweon et al. (2021) reported that stochastic processes played

450 a more important role in the planktonic bacterial communities in the River Thames,

451 presumably due to the influence of various natural and anthropogenic sources of the surface

452 water. What we found showed that in a water ecosystem almost isolated from other sources

453 than resource water, bacterial communities are predominantly shaped by deterministic abiotic

454 and biotic processes. The micro-eukaryotes, however, possess structurally and functionally 
455 more complex cells than prokaryotes (Massana and Logares, 2013), which could facilitate

456 niche adaptation and diminish the environmental filtering effect at the community level. This

457 was supported by our observation that the micro-eukaryotes were distributed with higher

458 occurrence frequency and lower variability across the canal compared to bacteria. The

459 different controlling mechanisms between bacterial and micro-eukaryotic community

460 assembly found here has been observed in other natural habitats, such as Antarctic (Logares et

461 al., 2018) and Tibetan lakes (Liu et al., 2020b). Our comparative analyses between bacteria

462 and micro-eukaryotes across canal sections and over seasons thus provide first informative

463 insights into the domain-specific, and space- and season-dependent patterns in the canal water

464 community assembly.

465 Divergent population lifestyles underly microbiota dynamics revealed by absolute

\section{6 quantification}

467 A synthesis of population and community ecological perspectives can provide valuable

468 insights into how microorganisms drive ecosystem processes (Koch et al., 2018). Microbial

469 populations in this giant system displayed interestingly divergent lifestyles reflecting the

470 difference in their adaptability to the canal environment. The Sloan neutral model sorted the

471 microbial ASVs to above-prediction, below-prediction (jointly non-neutral) and as-predicted

472 (neutral) groups, which were different in community composition (Fig. 5C and 5D),

473 indicating taxonomically dependent competitive fitness in the investigated canal. In addition,

474 we observed contrasting low $(\sim 20 \%)$ contribution of neutral group to community abundance 
475 despite their high proportion in richness $(\sim 80 \%)$ for both bacteria and micro-eukaryotes (Fig.

476 5B). Chen et al. (2019) reported for micro-eukaryotic communities in a subtropical river as

477 source of drinking water the dominance of neutral groups in both richness and abundance of

478 the overall communities as well as across taxonomic groups. These contrasting patterns

479 suggested that microorganisms are prone to more selective processes in an engineered canal

480 compared with natural habitats.

In particular, we demonstrate the use of local growth factor (LGF) as a meaningful

metric to infer growth of immigrating microbial taxa in the canal by employing absolute

484 bacterial and $82.3 \%$ of micro-eukaryotic populations were capable of sustaining growth

485 (LGF > 0), implying again better acclimation of eukaryotes to the environmental conditions in

486 the canal. It is worth clarifying that the microbial growth estimated here was aimed to

487 characterize fate of immigrating microorganisms across the canal, calculated based on relative

488 fluctuation in absolute abundance at local sites compared with canal head, and thus is not

489 equivalent to per-capita or population growth in the Sloan neutral model (Sloan et al., 2006).

490 Limnohabitans sp., ubiquitous in a wide variety of freshwater habitats, showed remarkable

491 growth across the canal. Members of Limnohabitans typically display high growth rate as

492 well as metabolic flexibility, and they actively utilize alga-derived dissolved organic

493 substances and are vulnerable to protist grazing (Horňák et al., 2017; Šimek et al., 2013;

494 Šimek et al., 2011). Cryptophyceae were among the micro-eukaryotes that displayed

495 prominent growth throughout the canal, which are small flagellates as important food of many 
496 protists and micro-crustaceans (Horňák et al., 2017; Posch et al., 2015), and are perceived as 497 characteristic of clean water (Lackey, 1941). Thus, Limnohabitans and Cryptophyceae are 498 potentially important constituents of water food webs in the investigated canal. Two 499 cyanobacterial lineages (Cyanobum PCC-6307) and Acinetobacter spp., on the contrary, were 500 clearly depressed in the canal downstream sections, demonstrating the elimination of these 501 microorganisms of water security concern along the canal. Acinetobacter spp. are ubiquitous 502 in nature of major ecological and clinical significance, featuring, for instance, 503 multi-antimicrobial resistance and rapid resistance development (Jung and Park, 2015). The

504 diminishing of the versatile Acinetobacter spp. along the canal could possibly be attributed to 505 unfavorable environmental conditions (Al Atrouni et al., 2016) or composite intra- and 506 inter-domain biological interactions. Further research efforts with genome-centric 507 metagenomic approaches are needed to elaborate the mechanisms behind such die-off patterns.

508 It is noteworthy that due to the restriction in taxonomic resolution of the ribosomal RNA gene 509 sequencing analysis employed in this study, species-level confirmation is still needed to 510 evaluate the relevance of these potentially pathogenic microorganisms. Together, our results 511 demonstrated the power of local growth factor to identify key players in the investigated 512 system and facilitate predicting fate of the microbes traversing such thousand-kilometer water 513 diversion canal.

\section{Conclusions}

515 The main canal of the Middle Route Project of the South-to-North Water Diversion projects is 
516 an engineered system where the aquatic ecosystem is still at its early forming stage (since

517 2014). Various aspects of hydrology, geochemistry and ecology need to be intricately

518 elaborated to jointly contribute to sustained system's eco-environmental services, especially

519 water auto-purification ensuring its quality and competence for designed social-economic

520 aims. The present study uncovered the underlying microbial community assembly

521 mechanisms and population dynamics in a giant engineered water diversion water canal, such

522 as the sharp decline of potentially harm-producing microbes (e.g., Cyanobacteria) and

523 potential pathogens (e.g., Acinetobacter spp.), and the favorable enrichment of alga-derived

524 organic scavengers (e.g., Limnohabitans) as potential key microbial food-web players,

525 demonstrating the valuable insights provided by microbial community and population

526 assessments for water monitoring and their potential in indicating ecosystem disturbance.

527 Furthermore, the bacterial community shift driven by combined environmental effects as well

528 as the stochasticity in micro-eukaryotic community assembly needs to be considered in water

529 quality monitoring along the canal, towards an appropriate microbe-based biomonitoring

530 which enables deeper understanding of processes behind water quality parameters.

531 Considering possibly occasional pollution into the canal water caused by, for example, traffic

532 accidents on highway bridges across the canal, and the central roles microorganisms play in

533 the water quality maintenance, future efforts are needed to explore the metabolic functions of

534 microbial community contributing to water purification (e.g., carbon, nitrogen, and

535 phosphorus removal) during diversion and sustaining water quality. 


\section{Acknowledgments}

537 We would like to thank Yuanzhen He, Guoqing Zhang, Zhipeng Zhang, Shengguang Yuan,

538 Aoxiang Fan and Qinglin Jia for the assistance in water sampling. This work was supported

539 by National Natural Science Foundation of China (Grant No. 51908467 and No. U2040210),

540 and Zhejiang Provincial Natural Science Foundation of China (Grant No. LQ20C030002). 


\section{References}

543 Al Atrouni, A., Joly-Guillou, M.-L., Hamze, M. and Kempf, M. 2016. Reservoirs of non-baumannii $544 \quad$ Acinetobacter species. Frontiers in Microbiology 7, 49.

545 Arrigo, K.R. 2005. Marine microorganisms and global nutrient cycles. Nature 437(7057), 349-355.

546

547

548

549

550

551

552

553

554

555

556

557

558

559

560

561

562

563

564

565

566

567

568

569

570

571

572

Barlow, J.T., Bogatyrev, S.R. and Ismagilov, R.F. 2020. A quantitative sequencing framework for absolute abundance measurements of mucosal and lumenal microbial communities. Nature Communications 11(1), 2590.

Battin, T.J., Besemer, K., Bengtsson, M.M., Romani, A.M. and Packmann, A.I. 2016. The ecology and biogeochemistry of stream biofilms. Nature Reviews Microbiology 14(4), 251-263.

Bell, T., Newman, J.A., Silverman, B.W., Turner, S.L. and Lilley, A.K. 2005. The contribution of species richness and composition to bacterial services. Nature 436(7054), 1157-1160.

Berry, D., Xi, C. and Raskin, L. 2006. Microbial ecology of drinking water distribution systems. Current Opinion in Biotechnology 17(3), 297-302.

Blanco-Bercial, L., Bradford-Grieve, J. and Bucklin, A. 2011. Molecular phylogeny of the Calanoida (Crustacea: Copepoda). Molecular Phylogenetics and Evolution 59(1), 103-113.

Bolyen, E., Rideout, J.R., Dillon, M.R., Bokulich, N.A., Abnet, C.C., Al-Ghalith, et al. 2019. Reproducible, interactive, scalable and extensible microbiome data science using QIIME 2. Nature Biotechnology 37, 852-857.

Borcard, D. and Legendre, P. 2002. All-scale spatial analysis of ecological data by means of principal coordinates of neighbour matrices. Ecological Modelling 153(1), 51-68.

Bradford-Grieve, J.M. 2002. Colonization of the pelagic realm by calanoid copepods. Hydrobiologia $485(1), 223-244$

Briones, A. and Raskin, L. 2003. Diversity and dynamics of microbial communities in engineered environments and their implications for process stability. Current Opinion in Biotechnology 14(3), 270-276.

Bru, D., Ramette, A., Saby, N.P.A., Dequiedt, S., Ranjard, L., Jolivet, C., Arrouays, D. and Philippot, L. 2011. Determinants of the distribution of nitrogen-cycling microbial communities at the landscape scale. The ISME Journal 5(3), 532-542.

Burns, A.R., Stephens, W.Z., Stagaman, K., Wong, S., Rawls, J.F., Guillemin, K. and Bohannan, B.J.M. 2016. Contribution of neutral processes to the assembly of gut microbial communities in the zebrafish over host development. The ISME Journal 10, 655. 
573

574

575

576

577

578

579

580

581

582

583

584

585

586

587

588

589

590

591

592

593

594

595

596

597

598

599

600

601

602

603

604

605

606

Callahan, B.J., McMurdie, P.J., Rosen, M.J., Han, A.W., Johnson, A.J.A. and Holmes, S.P. 2016. DADA2: High-resolution sample inference from Illumina amplicon data. Nature Methods 13(7), 581-583.

Chase, J.M. and Myers, J.A. 2011. Disentangling the importance of ecological niches from stochastic processes across scales. Philosophical Transactions of the Royal Society B: Biological Sciences 366(1576), 2351-2363.

Chen, F.Z., You, L.J., Yang, F., Wang, L.N., Guo, X.Q., Gao, F., Hua, C., Tan, C., Fang, L., Shan, R.Q., Zeng, W.J., Wang, B., Wang, R., Xu, X. and Wei, X.F. 2020. CNGBdb: China National GeneBank DataBase. Yi Chuan 42(8), 799-809.

Chen, W., Ren, K., Isabwe, A., Chen, H., Liu, M. and Yang, J. 2019. Stochastic processes shape microeukaryotic community assembly in a subtropical river across wet and dry seasons. Microbiome 7(1), 138.

Chen, Z., Yuan, J., Sun, F., Zhang, F., Chen, Y., Ding, C., Shi, J., Li, Y. and Yao, L. 2018. Planktonic fungal community structures and their relationship to water quality in the Danjiangkou Reservoir, China. Scientific Reports 8(1), 10596.

Dang, C., Liu, W., Lin, Y., Zheng, M., Jiang, H., Chen, Q. and Ni, J. 2018. Dominant role of ammonia-oxidizing bacteria in nitrification due to ammonia accumulation in sediments of Danjiangkou reservoir, China. Applied Microbiology and Biotechnology 102(7), 3399-3410.

Du, M., Peng, X., Zhang, H., Ye, C., Dasgupta, S., Li, J., Li, J., Liu, S., Xu, H., Chen, C., Jing, H., Xu, H., Liu, J., He, S., He, L, Cai, S., Chen, S. and Ni, J. 2021. Geology, environment, and life in the deepest part of the world's oceans. The Innovation 2(2), 100109.

Ghylin, T.W., Garcia, S.L., Moya, F., Oyserman, B.O., Schwientek, P., Forest, K.T., et al. 2014. Comparative single-cell genomics reveals potential ecological niches for the freshwater acl Actinobacteria lineage. The ISME Journal 8, 2503.

Glöckner, F.O., Zaichikov, E., Belkova, N., Denissova, L., Pernthaler, J., Pernthaler, A. and Amann, R. 2000. Comparative $16 S$ rRNA analysis of lake bacterioplankton reveals globally distributed phylogenetic clusters including an abundant group of Actinobacteria. Applied and Environmental Microbiology 66(11), 5053-5065.

Guo, X., Chen, F., Gao, F., Li, L., Liu, K., You, L., Hua, C., Yang, F., Liu, W., Peng, C., Wang, L., Yang, X., Zhou, F., Tong, J., Cai, J., Li, Z., Wan, B., Zhang, L., Yang, T., Zhang, M., Yang, L., Yang, Y., Zeng, W., Wang, B., Wei, X. and $\mathrm{Xu}, \mathrm{X}$. 2020. CNSA: a data repository for archiving omics data. Database (Oxford) 2020.

Gweon, H.S., Bowes, M.J., Moorhouse, H.L., Oliver, A.E., Bailey, M.J., Acreman, M.C. and Read, D.S. 2021. Contrasting community assembly processes structure lotic bacteria 
Hanson, C.A., Fuhrman, J.A., Horner-Devine, M.C. and Martiny, J.B.H. 2012. Beyond biogeographic patterns: processes shaping the microbial landscape. Nature Reviews Microbiology 10(7), 497-506.

Horňák, K., Kasalický, V., Šimek, K. and Grossart, H.P. 2017. Strain-specific consumption and transformation of alga-derived dissolved organic matter by members of the Limnohabitans- $C$ and Polynucleobacter-B clusters of Betaproteobacteria. Environmental Microbiology 19(11), 4519-4535.

Hu, A., Ju, F., Hou, L., Li, J., Yang, X., Wang, H., Mulla, S.I., Sun, Q., Bürgmann, H. and Yu, C.-P. 2017. Strong impact of anthropogenic contamination on the co-occurrence patterns of a riverine microbial community. Environmental Microbiology 19(12), 4993-5009.

618

Hull, N.M., Ling, F., Pinto, A.J., Albertsen, M., Jang, H.G., Hong, P.-Y., Konstantinidis, K.T., LeChevallier,

619 M., Colwell, R.R. and Liu, W.-T. 2019. Drinking water microbiome project: is it time? Trends in Microbiology 27(8), 670-677.

Ju, F., Beck, K., Yin, X., Maccagnan, A., McArdell, C.S., Singer, H.P., Johnson, D.R., Zhang, T. and Bürgmann, H. 2019. Wastewater treatment plant resistomes are shaped by bacterial composition, genetic exchange, and upregulated expression in the effluent microbiomes. The ISME Journal 13(2), 346-360.

Ju, F., Lau, F. and Zhang, T. 2017. Linking microbial community, environmental variables, and methanogenesis in anaerobic biogas digesters of chemically enhanced primary treatment sludge. Environmental Science and Technology 51(7), 3982-3992.

Ju, F., Xia, Y., Guo, F., Wang, Z. and Zhang, T. 2014. Taxonomic relatedness shapes bacterial assembly in activated sludge of globally distributed wastewater treatment plants. Environmental Microbiology 16(8), 2421-2432.

$631 \mathrm{Ju}, \mathrm{F}$. and Zhang, T. 2015. Bacterial assembly and temporal dynamics in activated sludge of a full-scale municipal wastewater treatment plant. The ISME journal 9(3), 683. microbiology: current state and perspectives. Applied Microbiology and Biotechnology 99, 2533-2548.

Keshri, J., Pradeep Ram, A.S., Nana, P.A. and Sime-Ngando, T. 2018. Taxonomical resolution and distribution of bacterioplankton along the vertical gradient reveals pronounced spatiotemporal patterns in contrasted temperate freshwater lakes. Microbial Ecology 76(2), 
640

641

642

643

644

645

646

647

648

649

650

651

652

653

654

655

656

657

658

659

660

661

662

663

664

665

666

667

668

669

670

671

672

673

Koch, B.J., McHugh, T.A., Hayer, M., Schwartz, E., Blazewicz, S.J., Dijkstra, P., van Gestel, N., Marks, J.C., Mau, R.L., Morrissey, E.M., Pett-Ridge, J. and Hungate, B.A. 2018. Estimating taxon-specific population dynamics in diverse microbial communities. Ecosphere 9(1), e02090.

Lackey, J.B. 1941. Two groups of flagellated algae serving as indicators of clean water. Journal (American Water Works Association) 33(6), 1099-1110.

Li, Y.-Y., Zhang, Z., Yin, W., Hu, L., Liang, Z., Wang, F. and Lei, G. 2012. Zooplankton diversity in the water resource area of the Mid-Line Project of South to North Water Division. Advanced Materials Research 356-360, 199-203.

Liao, Y., Chen, J., Brandt, B.W., Zhu, Y., Li, J., van Loveren, C. and Deng, D.M. 2015. Identification and functional analysis of genome mutations in a fluoride-resistant Streptococcus mutans strain. PLOS ONE 10(4), e0122630.

Lindh, M.V., Lefébure, R., Degerman, R., Lundin, D., Andersson, A. and Pinhassi, J. 2015. Consequences of increased terrestrial dissolved organic matter and temperature on bacterioplankton community composition during a Baltic Sea mesocosm experiment. AMBIO $44(3), 402-412$.

Liu, H., Yin, J. and Feng, L. 2018a. The dynamic changes in the storage of the Danjiangkou Reservoir and the influence of the South-North Water Transfer Project. Scientific Reports 8(1), 8710.

Liu, J., Zhu, S., Liu, X., Yao, P., Ge, T. and Zhang, X.-H. 2020a. Spatiotemporal dynamics of the archaeal community in coastal sediments: assembly process and co-occurrence relationship. The ISME Journal 14(6):1463-1478.

Liu, K., Liu, Y., Hu, A., Wang, F., Chen, Y., Gu, Z., Anslan, S. and Hou, J. 2020b. Different community assembly mechanisms underlie similar biogeography of bacteria and microeukaryotes in Tibetan lakes. FEMS Microbiology Ecology 96(6).

Liu, T., Zhang, A.N., Wang, J., Liu, S., Jiang, X., Dang, C., Ma, T., Liu, S., Chen, Q., Xie, S., Zhang, T. and Ni, J. 2018b. Integrated biogeography of planktonic and sedimentary bacterial communities in the Yangtze River. Microbiome 6(1), 16.

Logares, R., Tesson, S.V.M., Canbäck, B., Pontarp, M., Hedlund, K. and Rengefors, K. 2018. Contrasting prevalence of selection and drift in the community structuring of bacteria and microbial eukaryotes. Environmental Microbiology 20(6), 2231-2240.

Luo, Z., Li, S., Hou, K. and Ji, G. 2019. Spatial and seasonal bacterioplankton community dynamics in the main channel of the Middle Route of South-to-North Water Diversion Project. Research in Microbiology 170(1), 24-34. 
674 Massana, R. and Logares, R. 2013. Eukaryotic versus prokaryotic marine picoplankton ecology. $675 \quad$ Environmental Microbiology 15(5), 1254-1261.

676 Mei, R., Kim, J., Wilson, F.P., Bocher, B.T.W. and Liu, W.-T. 2019. Coupling growth kinetics modeling

677

678

679

680

681

682

683

684

685

686

687

688

689

690

691

692

693

694

695

696

697

698

699

700

701

702

703

704

705 with machine learning reveals microbial immigration impacts and identifies key environmental parameters in a biological wastewater treatment process. Microbiome $7(1)$, 65.

Mei, R. and Liu, W.-T. 2019. Quantifying the contribution of microbial immigration in engineered water systems. Microbiome 7(1), 144.

Ning, D., Deng, Y., Tiedje, J.M. and Zhou, J. 2019. A general framework for quantitatively assessing ecological stochasticity. Proceedings of the National Academy of Sciences 116(34), 16892-16898.

Niderdorfer, R., Fragner, L., Yuan, L., Hausherr, D., Wei, J., Magyar, P., Joss, A., Lehmann, M.F., Ju, F. and Bürgmann, H. 2021. Distinct growth stages controlled by the interplay of deterministic and stochastic processes in functional anammox biofilms. Water Research 200, 117225.

Nong, X., Shao, D., Xiao, Y. and Zhong, H. 2019. Spatio-temporal characterization analysis and water quality assessment of the South-to-North Water Diversion Project of China. International Journal Environmental Research and Public Health 16(12), 2227.

Ochoa-Herrera, V., Banihani, Q., León, G., Khatri, C., Field, J.A. and Sierra-Alvarez, R. 2009. Toxicity of fluoride to microorganisms in biological wastewater treatment systems. Water Research 43(13), 3177-3186.

Posch, T., Eugster, B., Pomati, F., Pernthaler, J., Pitsch, G. and Eckert, E. 2015. Network of interactions between ciliates and phytoplankton during spring. Frontiers in Microbiology 6 , 14.

Pu, W., Quan, W., Ma, Z., Shi, X., Zhao, X., Zhang, L., Wang, Z. and Wang, W. 2017. Long-term trend of chemical composition of atmospheric precipitation at a regional background station in Northern China. Science of The Total Environment 580,1340-1350.

R Core Team 2020 R: A language and environment for statistical computing, $R$ foundation for statistical computing.

Sagova-Mareckova, M., Boenigk, J., Bouchez, A., Cermakova, K., Chonova, T., Cordier, et al. 2021. Expanding ecological assessment by integrating microorganisms into routine freshwater biomonitoring. Water Research 191, 116767.

Šimek, K., Kasalický, V., Jezbera, J., Horňák, K., Nedoma, J., Hahn, M.W., Bass, D., Jost, S. and Boenigk, J. 
706

707

708

709

710

711

712

713

714

715

716

717

718

719

720

721

722

723

724

725

726

727

728

729

730

731

732

733

734

735

736

737

2013. Differential freshwater flagellate community response to bacterial food quality with a focus on Limnohabitans bacteria. The ISME Journal 7(8), 1519-1530.

Šimek, K., Kasalický, V., Zapomělová, E. and Horňák, K. 2011. Alga-derived substrates select for distinct betaproteobacterial lineages and contribute to niche separation in Limnohabitans strains. Applied and Environmental Microbiology 77(20), 7307-7315.

Sloan, W.T., Lunn, M., Woodcock, S., Head, I.M., Nee, S. and Curtis, T.P. 2006. Quantifying the roles of immigration and chance in shaping prokaryote community structure. Environmental Microbiology 8(4), 732-740.

Stämmler, F., Gläsner, J., Hiergeist, A., Holler, E., Weber, D., Oefner, P.J., Gessner, A. and Spang, R. 2016. Adjusting microbiome profiles for differences in microbial load by spike-in bacteria. Microbiome 4(1), 28.

Stegen, J.C., Lin, X., Fredrickson, J.K., Chen, X., Kennedy, D.W., Murray, C.J., Rockhold, M.L. and Konopka, A. 2013. Quantifying community assembly processes and identifying features that impose them. The ISME Journal 7, 2069.

Tkacz, A., Hortala, M. and Poole, P.S. 2018. Absolute quantitation of microbiota abundance in environmental samples. Microbiome 6(1), 110.

Vanwonterghem, I., Jensen, P.D., Dennis, P.G., Hugenholtz, P., Rabaey, K. and Tyson, G.W. 2014. Deterministic processes guide long-term synchronised population dynamics in replicate anaerobic digesters. The ISME Journal 8(10), 2015-2028.

Vellend, M. and Agrawal, A. 2010. Conceptual synthesis in community ecology. The Quarterly Review of Biology 85(2), 183-206.

Vieira-Silva, S., Sabino, J., Valles-Colomer, M., Falony, G., Kathagen, G., Caenepeel, C., Cleynen, I., van der Merwe, S., Vermeire, S. and Raes, J. 2019. Quantitative microbiome profiling disentangles inflammation- and bile duct obstruction-associated microbiota alterations across PSC/IBD diagnoses. Nature Microbiology 4(11), 1826-1831.

Wang, W., Ren, K., Chen, H., Gao, X., Rønn, R. and Yang, J. 2020. Seven-year dynamics of testate amoeba communities driven more by stochastic than deterministic processes in two subtropical reservoirs. Water Research 185, 116232.

Woolhouse, M. E. J., Gowtage-Sequeria, S. and Evans, B. 2015. Quantitative analysis of the characteristics of emerging and re-emerging human pathogens. http://webarchive.nationalarchives.gov.uk/20121206154522/http://www.bis.gov.uk/assets/f oresight/docs/infectious-diseases/t16.pdf 2015. 
738

739

740

741

742

743

744

745

746

747

748

749

750

751

752

753

754

755

756
Wu, L., Ning, D., Zhang, B., Li, Y., Zhang, P., Shan, et al. 2019. Global diversity and biogeography of bacterial communities in wastewater treatment plants. Nature Microbiology 4, 1183-1195.

Yan, L., Herrmann, M., Kampe, B., Lehmann, R., Totsche, K.U. and Küsel, K. 2020. Environmental selection shapes the formation of near-surface groundwater microbiomes. Water Research $170,115341$.

Yang, C., Wang, Q., Simon, P.N., Liu, J., Liu, L., Dai, X., Zhang, X., Kuang, J., Igarashi, Y., Pan, X. and Luo, F. 2017. Distinct network interactions in particle-associated and free-living bacterial communities during a Microcystis aeruginosa bloom in a plateau lake. Frontiers in microbiology 8, 1202-1202.

Zhang, X., Gao, X., Li, C., Luo, X. and Wang, Y. 2019a. Fluoride contributes to the shaping of microbial community in high fluoride groundwater in Qiji County, Yuncheng City, China. Scientific Reports 9, 14488.

Zhang, X., Li, B., Xu, H., Wells, M., Tefsen, B. and Qin, B. 2019b. Effect of micronutrients on algae in different regions of Taihu, a large, spatially diverse, hypereutrophic lake. Water Research 151, 500-514.

Zhou, J., Deng, Y., Zhang, P., Xue, K., Liang, Y., Van Nostrand, J.D., Yang, Y., He, Z., Wu, L., Stahl, D.A., Hazen, T.C., Tiedje, J.M. and Arkin, A.P. 2014. Stochasticity, succession, and environmental perturbations in a fluidic ecosystem. Proceedings of the National Academy of Sciences 111(9), E836-E845. 


\section{$758 \quad$ Figure Legends}

759 Fig. 1 Four sampling campaigns at 19 sites along the $1432-\mathrm{km}$ main canal of the MRP of the

760 SNWD project selected for microbial and physicochemical analyses. Colored fans indicate

761 months of sampling at each site. The map was created using QGIS (version 3.10.5,

762 www.qgis.org).

763 Fig. 2 Seasonal variation of bacterial and micro-eukaryotic communities in the canal. (A-F)

764 Ribosomal RNA gene copies (A and B), Chao1 richness (C and D) and Shannon diversity

765 index (E and F) across seasons. Different letters above boxes indicate statistically significant

766 differences between months of sampling (Mann-Whitney $U$ test with the Benjamini-Hochberg

767 correction, $P<0.05)$. (G-H) Ordination analysis of communities using non-metric

768 multidimensional scaling (NMDS) based on Bray-Curtis dissimilarities. Trajectories indicate

769 locations of sampling sites along the canal.

770 Fig. 3 Microbial community composition and diversity along the MRP main canal $(\sim 1432 \mathrm{~km})$

771 of the SNWD project in October 2018. (A) Bacterial composition with abundant phyla (> 1\%)

772 and 10 most abundant genera shown. (B) Micro-eukaryotic composition with abundant phyla

$773(>1 \%)$ and 10 most abundant orders shown.

774 Fig. 4 Redundancy analysis (RDA) and variation partitioning analysis (VPA) demonstrating

775 the relative influence of seasonality, spatial and environmental factors on the canal microbial

776 communities. The RDA biplots show factors impacting bacterial (A) and micro-eukaryotic (B)

777 communities. The VPA diagrams show the percentage contribution of seasonal, spatial and 
778 environmental factors to bacterial (C) and micro-eukaryotic (D) community variations. Only

779 those factors identified to significantly influence bacterial and micro-eukaryotic communities

780 are shown in the RDA and included in the VPA analyses. PCNMs, geographic factors

781 generated by principal coordinate analysis of neighborhood matrices. T, temperature; F,

782 fluoride; TN, total nitrogen; DO, dissolved oxygen.

783 Fig. 5 Normalized stochasticity ratio (NST) estimation and microbiota partitioning by

784 population compliance to the neutral model prediction. (A) NST estimated stochasticity in

785 bacterial and micro-eukaryotic community assembly. (B) Proportions in richness (ASV

786 number) and abundance (sequence number) of the three fitting groups (occurrence frequency

787 above prediction, as predicted and below prediction) within the microbial communities to the

788 Sloan neutral model. (C and D) Taxonomic composition of the three fitting partitions in

789 richness (Ri) and abundance (Ab) for bacterial (C) and micro-eukaryotic (D) communities.

790 Only abundant bacterial phyla (> 1\%) and 10 most abundant micro-eukaryotic orders are

791 shown.

792 Fig. 6 Microbial population proliferation across canal sections evaluated by local growth

793 factor analysis. Distribution patterns of population local growth factors in the different canal

794 sections are shown with violin plots for bacteria (A) and micro-eukaryotes (B). Each dot

795 represents an ASV with colors indicating its average abundance across the canal. 


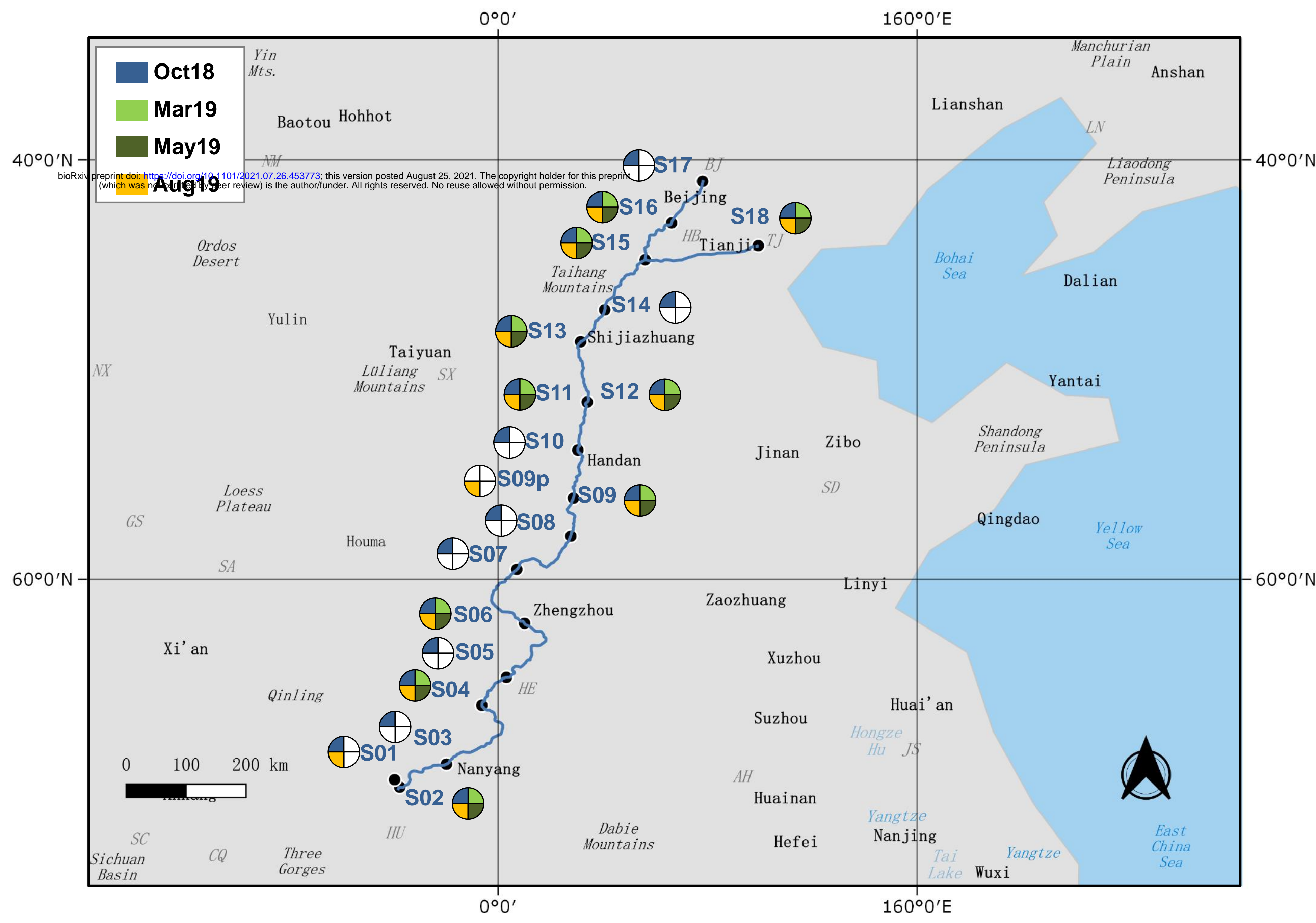




\section{Bacteria}
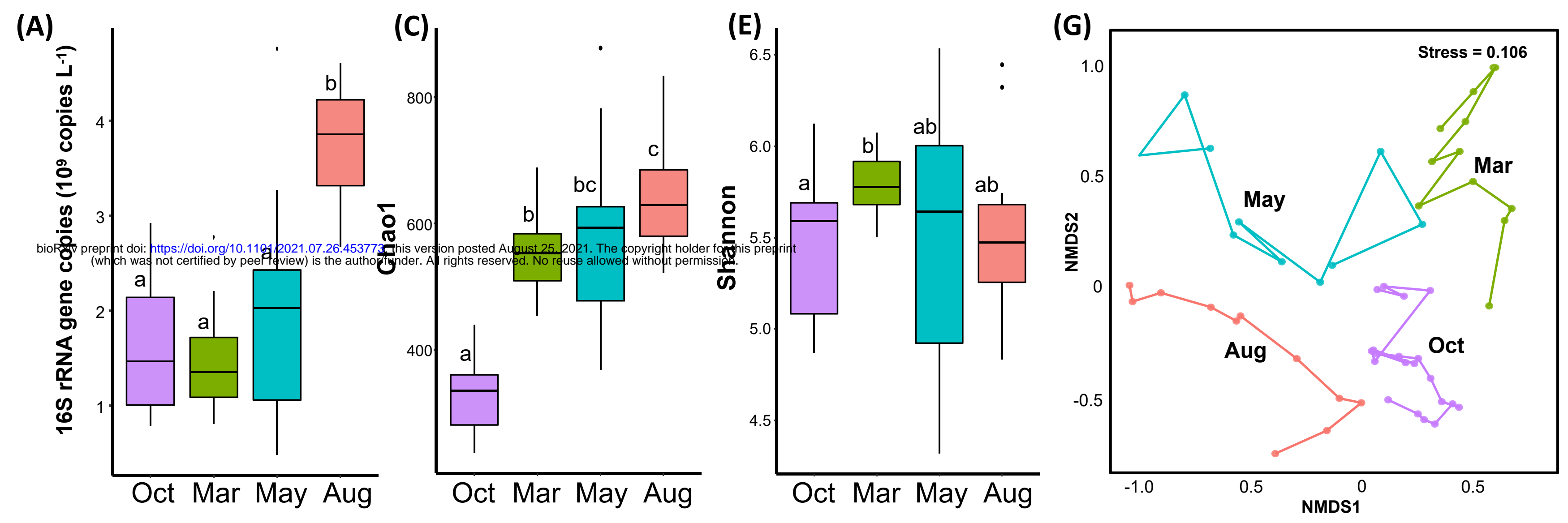

\section{Micro-eukaryotes}
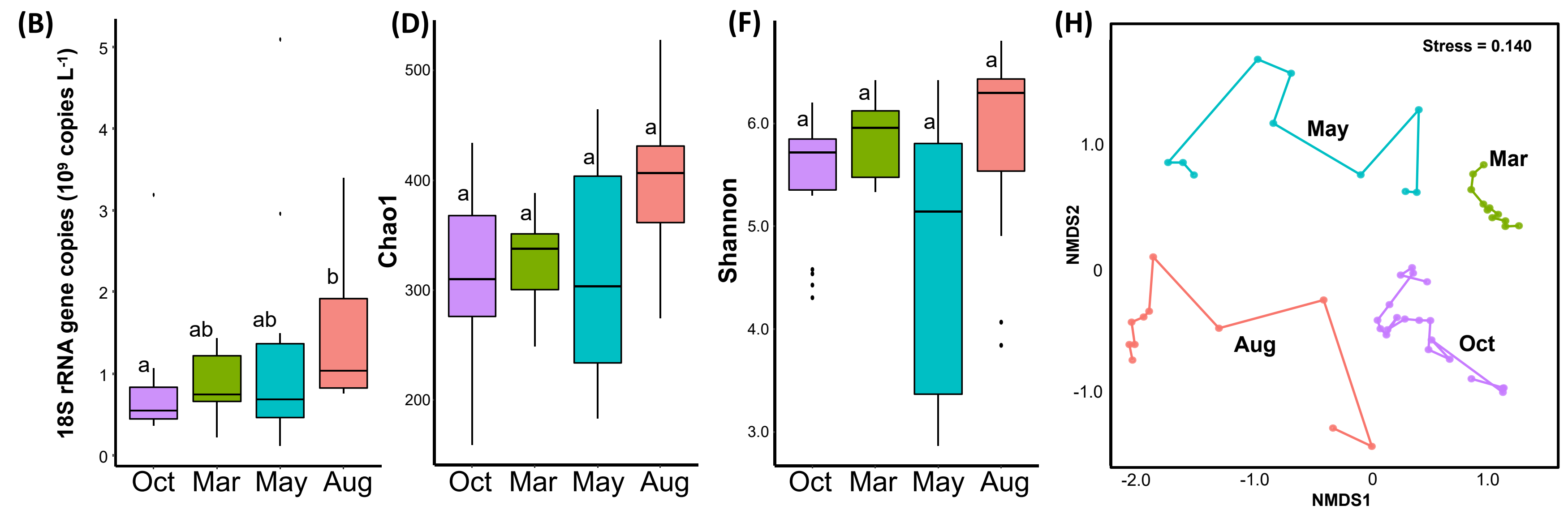


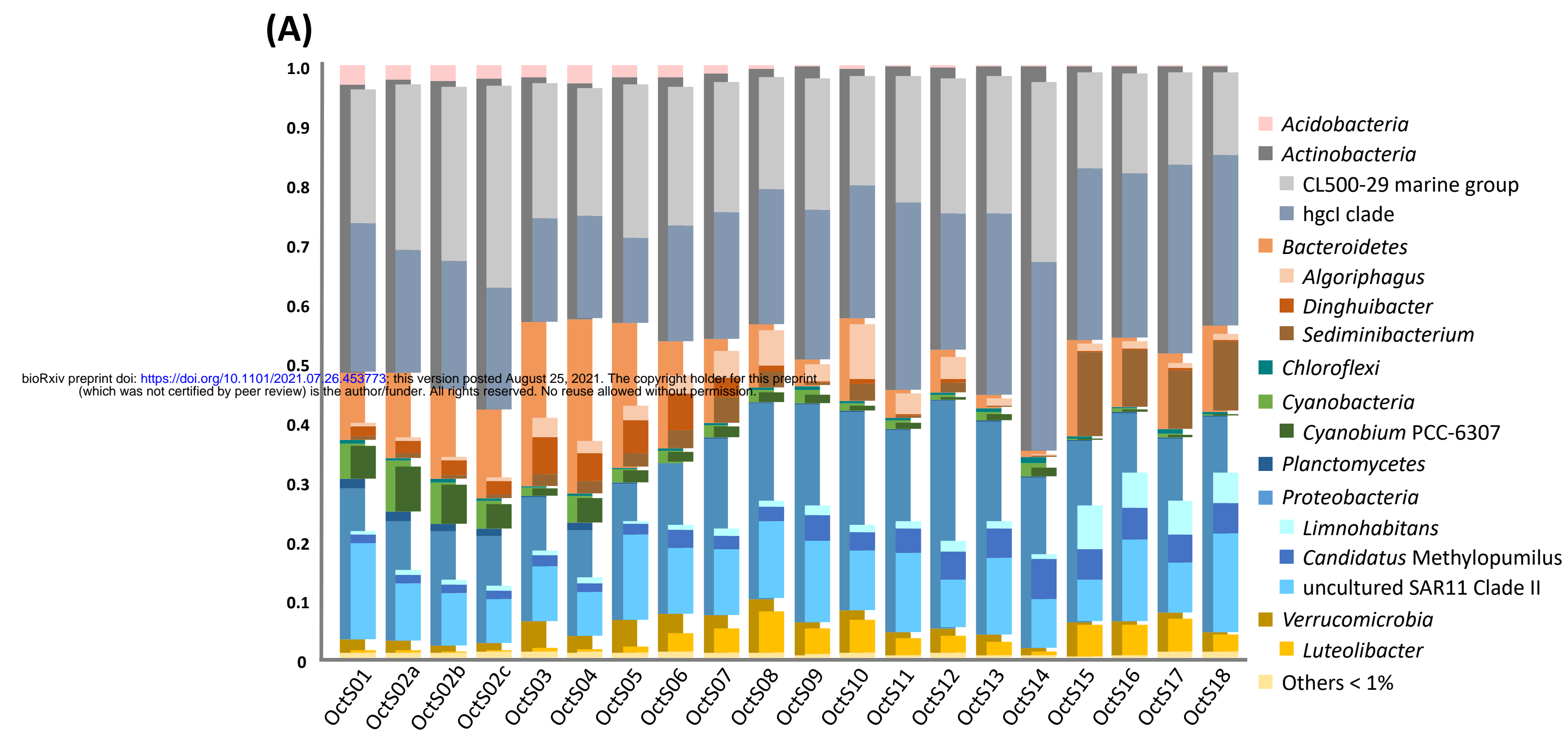

(B)

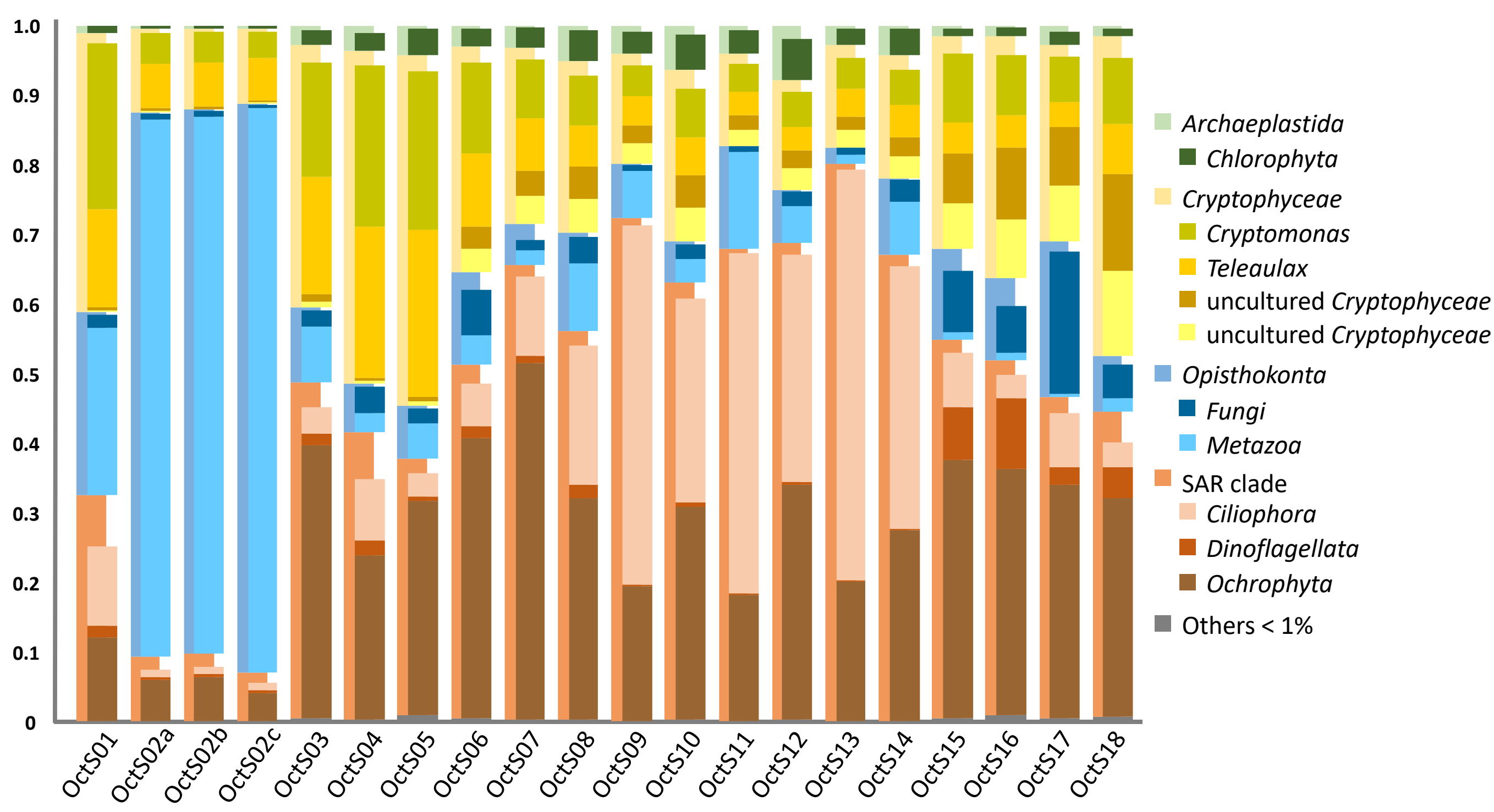


(A)

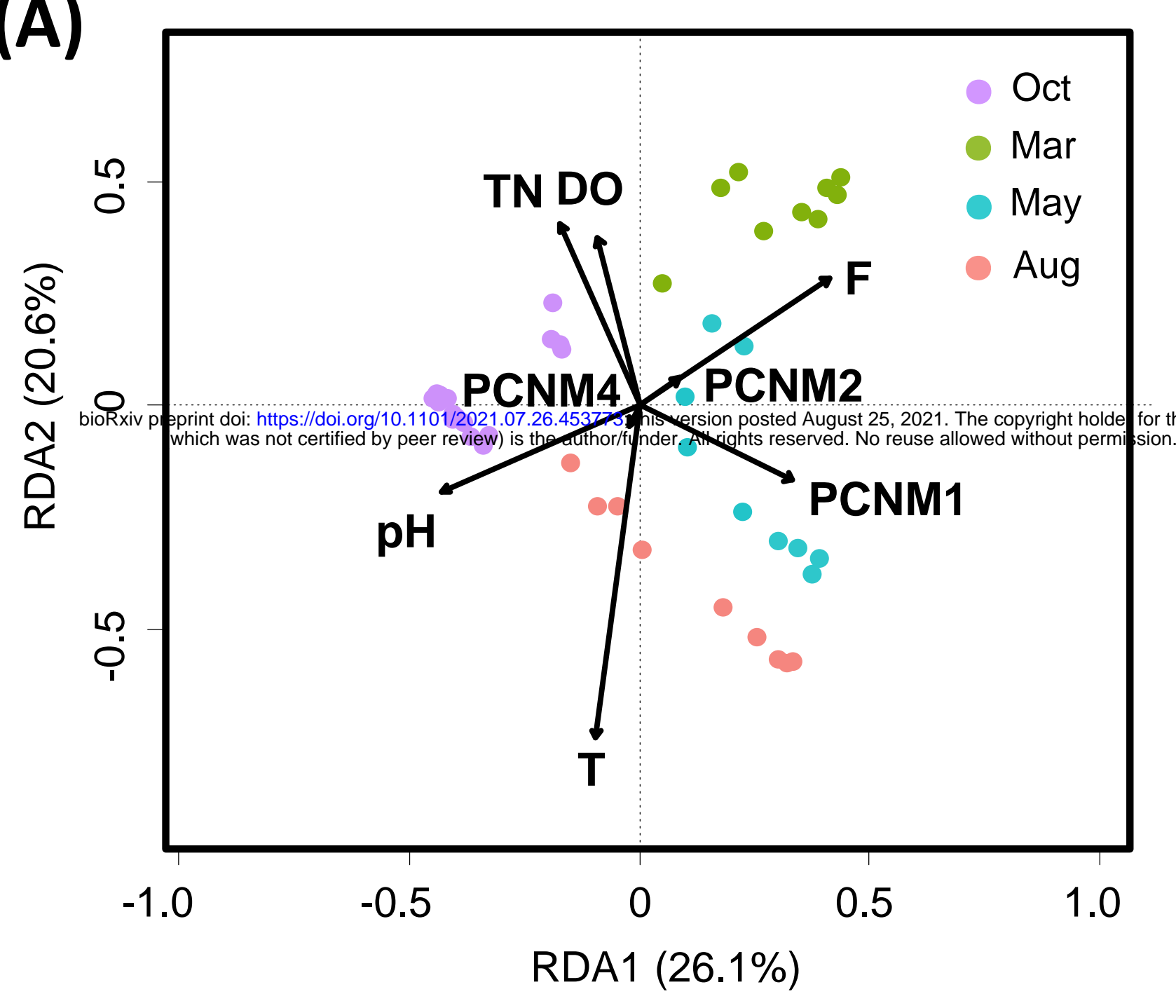

(C)

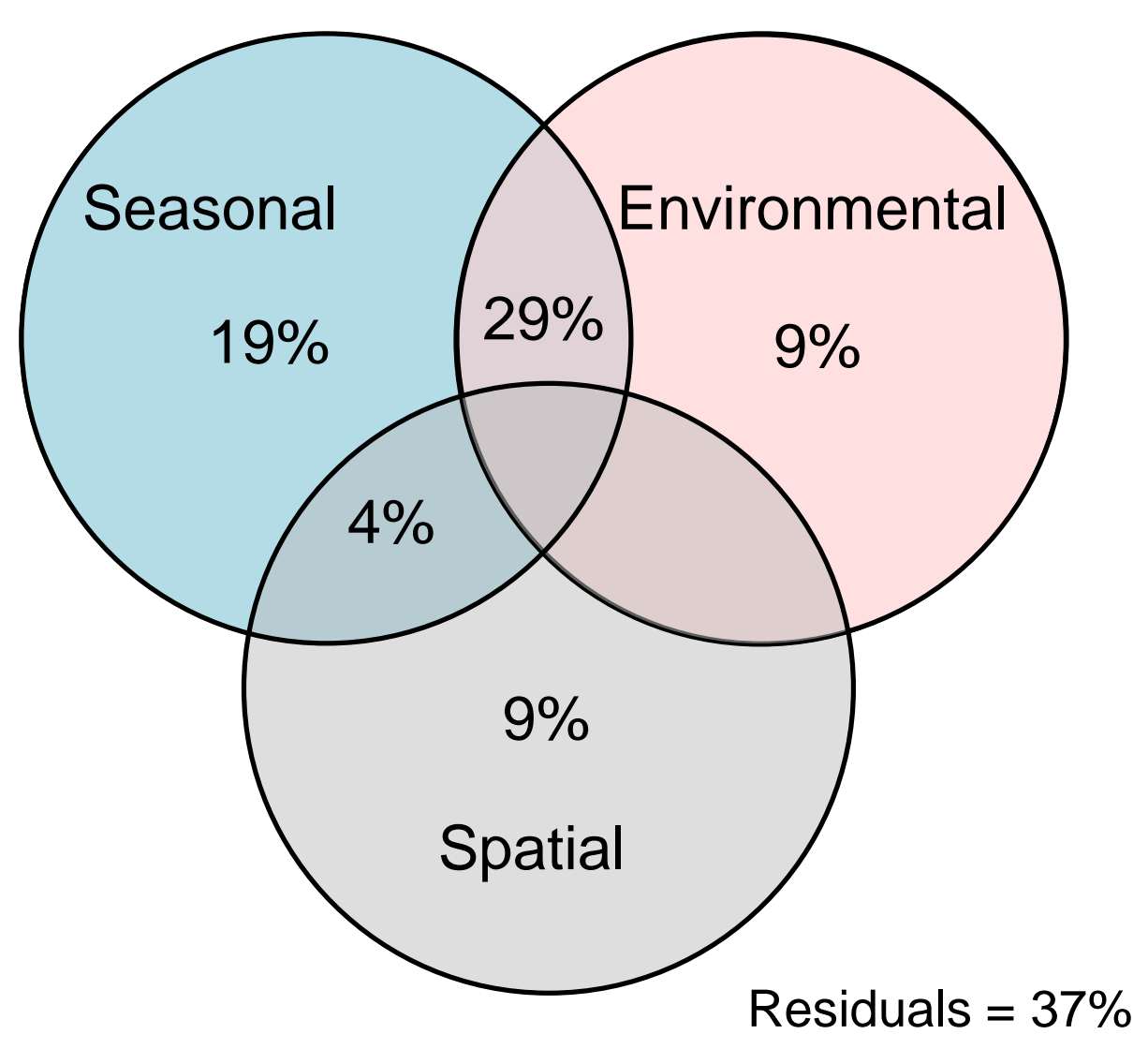

(B)

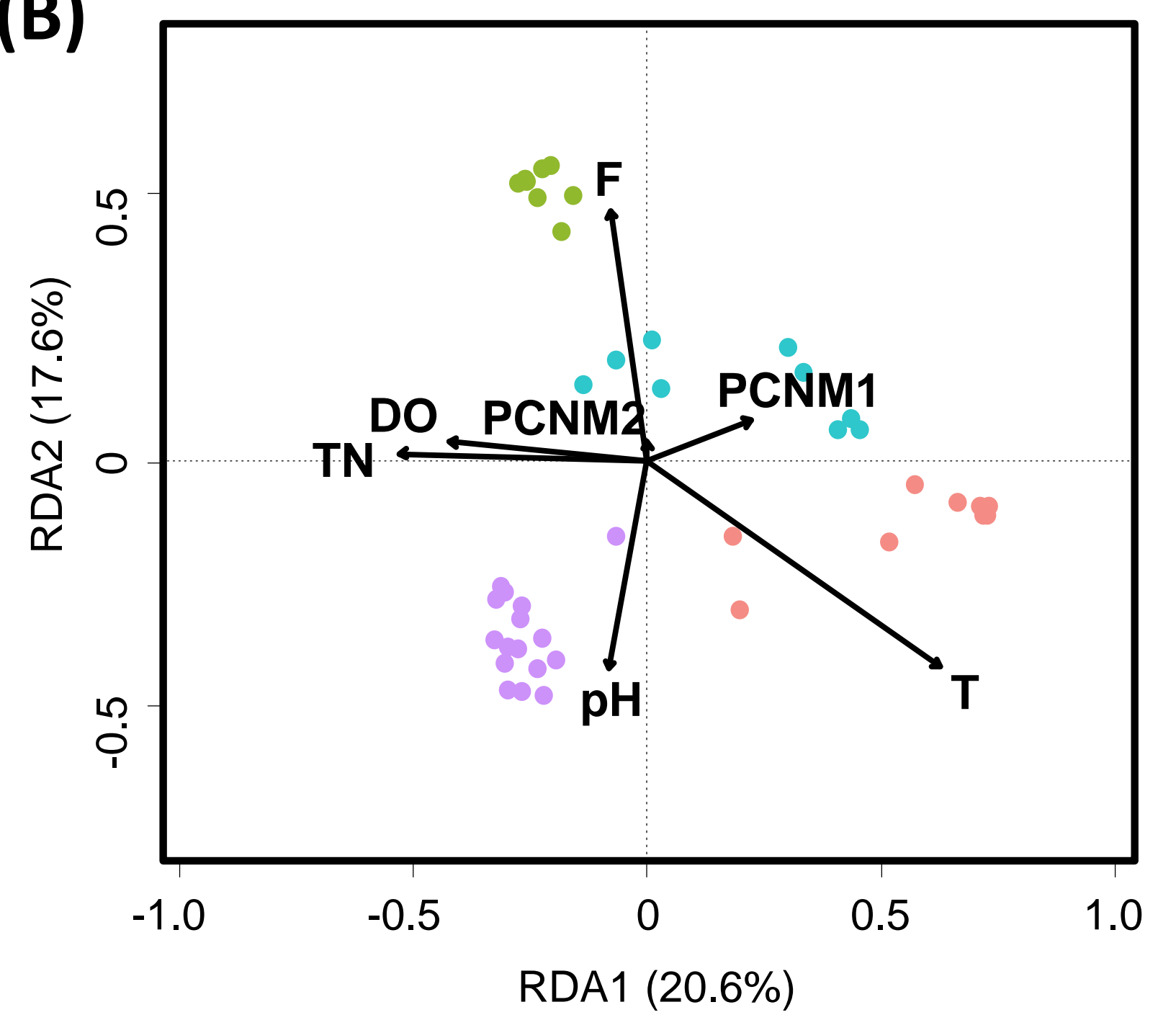

(D)

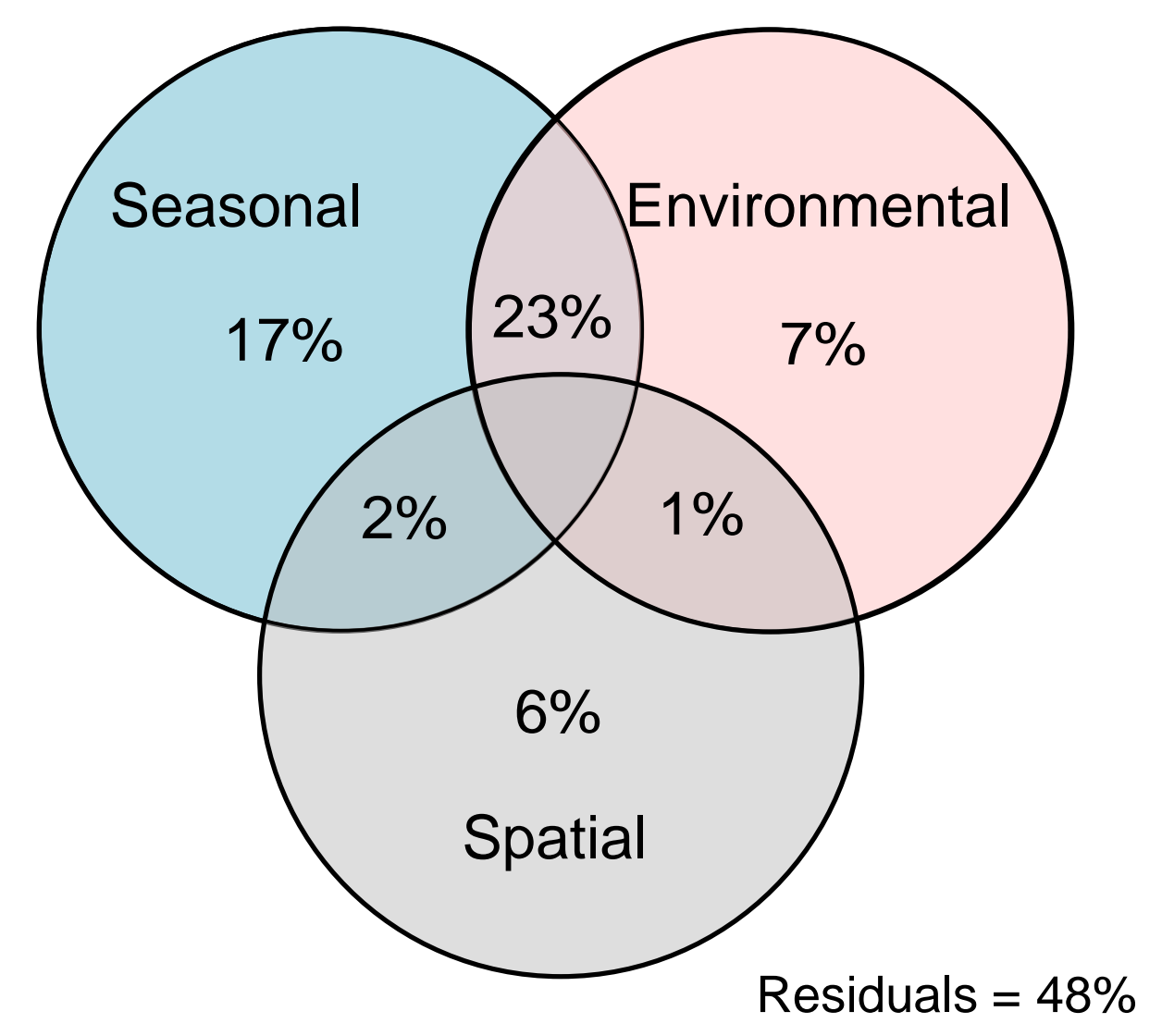



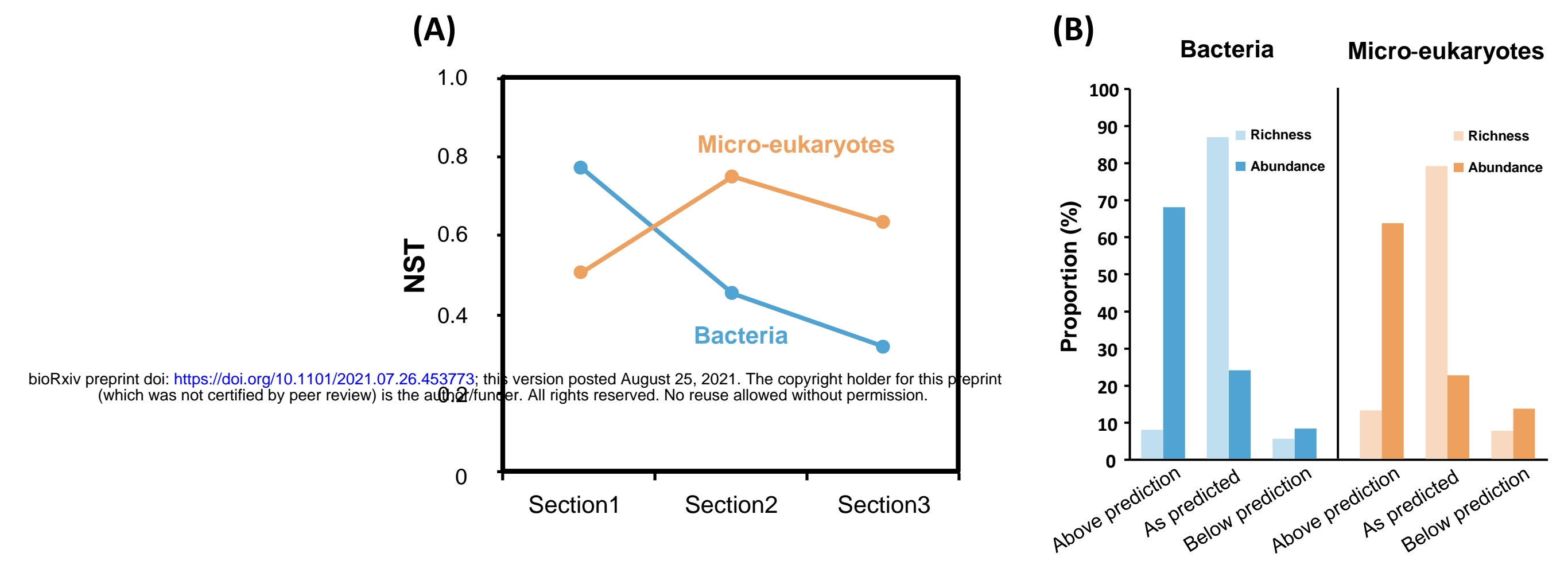

(C)

Proportion (\%)

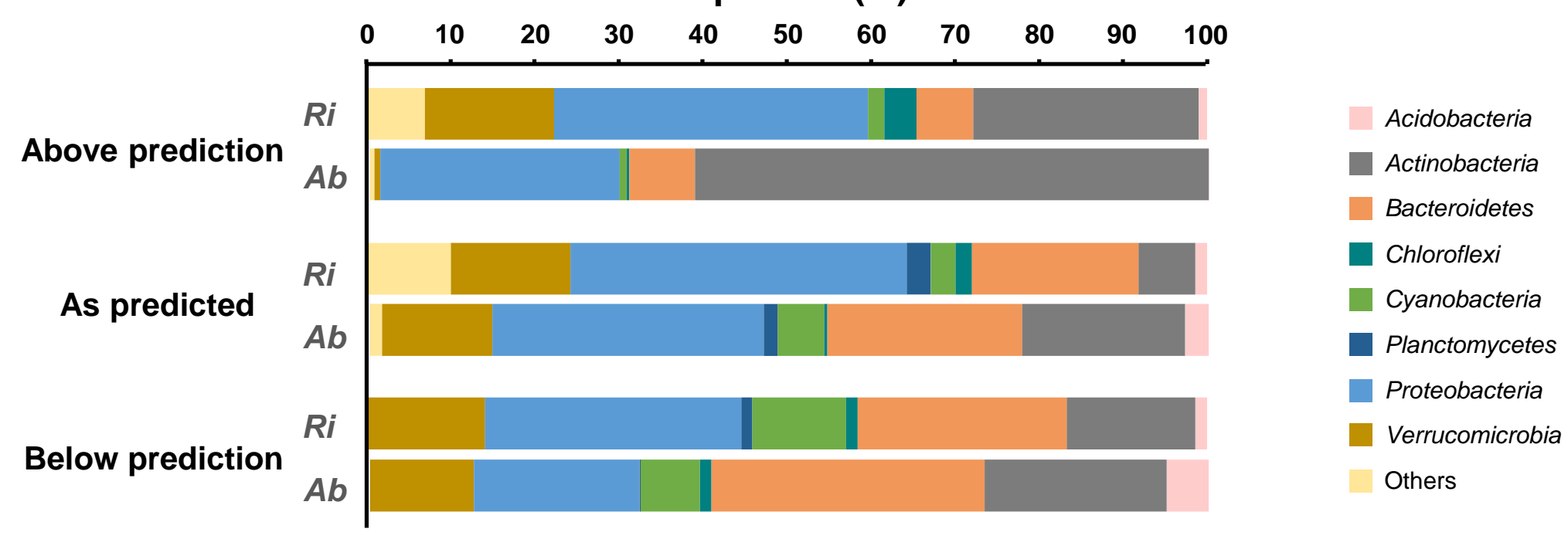

(D)

Proportion (\%)

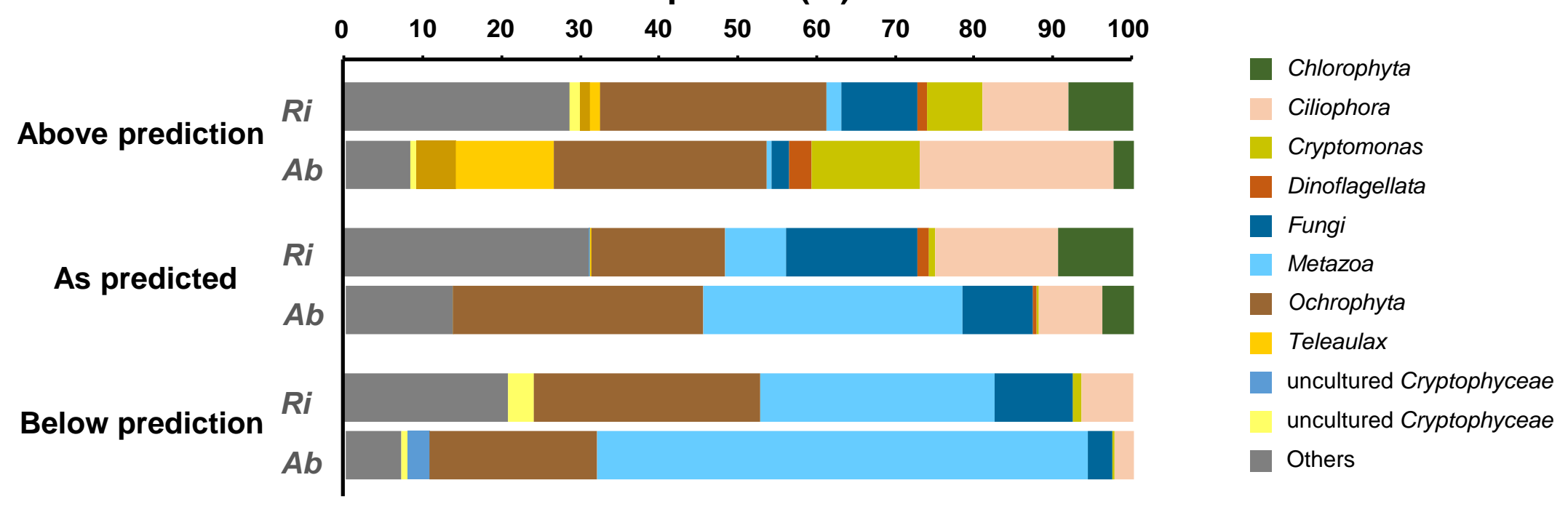


